\title{
Using thermography to investigate thermal characteristics of porous silicon
}

\author{
Adrian Keating ${ }^{* 1}$, Brad McNiven ${ }^{2}$, Xiao Sun ${ }^{1}$ and G. Todd Andrews ${ }^{2}$ \\ ${ }^{1}$ Department of Mechanical and Chemical Engineering, University of Western Australia, 35 Stirling Hwy, Crawley, Western \\ Australia 6009, Australia \\ ${ }^{2}$ Department of Physics and Physical Oceanography, Memorial University, St. John's, NL, Canada A1B 3X7 \\ * adrian.keating@uwa.edu.au
}

A low cost thermography system capable of submilliKelvin resolution has been developed and will be described through this work. The instrument is applied to the study of low thermal conductivity porous silicon films, which is a challenge given that thermography has previously been developed to study only thin (released) films or highly conducting films on insulating substrates. Our preliminary results indicate that the system is capable of measuring porous silicon thin films on highly conducting substrates, but requires application of the pertinent underlying physics for parameter determination.

\section{Introduction}

Laser writing is seen as an important tool to create functional structures such as waveguides [1] and diffraction gratings [2] in porous films which are traditionally difficult to process through traditional microfabrication techniques [3]. It is known that laser writing into porous films can be used to oxide regions of the film and hence the thermal properties are expected to change significantly [2]. However there have been no reports characterising the change in the films thermal properties due to local laser irradiation - previous studies have focused only on thermal measurement by a laser before or after the entire film is oxidation [4]. Here we consider a thermography approach to measure the thermal properties of porous silicon irradiated by a focused laser.

Thermography is a powerful technique to extract thermal and electrical characteristics from materials [5]. The optical system under consideration is illustrated in Figure 1. Here the external heat generator used is a laser modulated at frequency $f$, however electrical modulation of deposited contacts can also be used to locally heat the material. A long wavelength thermal imaging camera and lock-in techniques are used to capture the thermal emission from the surface. The lock-in approach multiplies each image frame by in-phase (sine) and quadrature (cosine) terms at the same frequency $(f)$ at which the external heat source is modulated. While traditionally expensive due to the cost of the thermal imaging camera and lasers, new low resolution thermal imagers (FLIR Lepton, $80 \times 60$ pixels, $20 \mathrm{mK}$ at 9 f.p.s) and inexpensive 405-nm laser diodes provide a path to construct this powerful tool at a fraction of traditional costs. We have combined these technologies with readily available low cost microcontrollers (Raspberry Pis and Arduinos) which can drive the laser, synchronise the thermal images and control a 2-axis motion stage with $30 \mu \mathrm{m}$ step accuracy. The physical system is contained within a $20 \times 20 \times 13 \mathrm{~cm}^{3}$ IP66 rated enclosure with gas inlet/outlets, permitting gas flow to subsequently investigate the effect of selected gases on the thermal properties of porous silicon films (not studied in this work). The system permits control of laser power, modulation rate

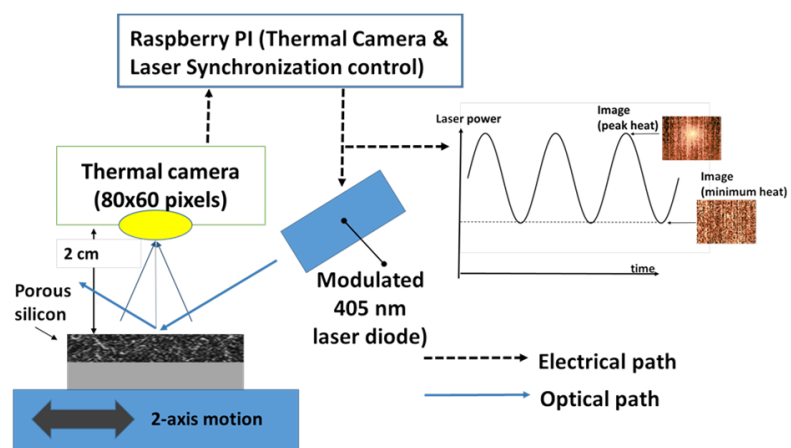

Figure 1: Optical system diagram for the thermography system developed as part of this work to investigate thermal characteristics of porous silicon. The physical housing of the system within a $20 \times 20 \times 13 \mathrm{~cm}^{3}$ IP66 rated enclosure.

and amplitude, gas flow and sample spatial location to provide a multi-dimensional space to map the thermal characteristics of porous films. The small size of the instrument allows easy transport to/from our collaborators (WA $\leftrightarrow$ Newfoundland) and direct network access to the instrument (telepresence) enables us to link together our remote researchers to collect data and update image-based firmware/software. A key feature of this image based lockin technique is that for every $180^{\circ}$ of the applied sine wave (see Figure 1), the image is effectively subtracted from itself so that common mode noise is eliminated and only the differential (modulated) heat source is detected. Averaging over a sufficiently large number of frames allows a measurement resolution well below the level at which the thermal camera is specified. The entire system costs less than AUD\$1000 in parts to assemble.

\section{Results and discussion}

Our preliminary goal was to determine if this measurement approach is applicable to porous silicon samples having a thin highly insulting layer (thermal conductivity as low as $0.05 \mathrm{~W} / \mathrm{m} . \mathrm{K}$ [6]) on top of a highly thermal conductive substrate (silicon thermal conductivity $148 \mathrm{~W} / \mathrm{m} . \mathrm{K}$ [7]). The porous films were anodised in a $15 \%$ HF/ethanol solution, on moderately doped p-type (100) silicon wafers with resistivity of $0.08-0.12 \Omega \cdot \mathrm{cm}$. The current density during anodisation was constant at $10 \mathrm{~mA} / \mathrm{cm}^{2}$ and the resulting film had a thickness of $t=2.7 \mu \mathrm{m}$ and an average porosity of $P=84 \%$.

The sample of porous silicon was heated by modulating the laser ( $1 \mathrm{~mW}$, spot size $0.5 \mathrm{~mm})$ at a fixed frequency, $f$. The elliptical beam of the laser was aligned with the major axis in the plane of incidence, so that at an incident angle of $45^{\circ}$, the beam shape was circular on the sample. The number of time averages was set to 100 periods of the sinewave, ensuring the cut-off frequency for the digital lock-in low-pass filter was $1 \%$ of the lock-in frequency, sufficient 
to supress high frequency noise. After each temporal average, the measurement cycle was resynchronized and a set of 4 (ensemble) averages were taken for each measurement at frequency, $f$. The measurement was repeated over a range from $0.5 \mathrm{~Hz}<f<1.2 \mathrm{~Hz}$ as the thermal diffusion length $\left(L_{D}\right)$ is expected to vary with modulation frequency [8]. A typical image showing the magnitude of the signal at a modulation frequency of $f=0.5 \mathrm{~Hz}$ is shown in Figure 2a. The thermographic measurement yields both magnitude (Figure 2b) and phase (Figure 2b) from the inphase and quadrature lock-in signal. The measurement over a field of view of $16 \times 12 \mathrm{~mm}^{2}$ clearly shows an increase in temperature of $23 \mathrm{mK}$ in the centre of the sample originating from the modulated optical beam. Under these low-pass and averaging conditions, the thermal resolution was $1 \mathrm{mK}$, however sub-mK resolution has been achieved with longer averaging.

Beyond the time and ensemble averaging of the signal, we also used the symmetry of the radial dependence of the thermography image to improve the signal to noise ratio. Figure 3 shows a radial histogram extracted from the magnitude and phase data shown in Figure 3. After radial spatial averaging, the single-sided radial distribution of temperature magnitude had a high signal to noise ratio, sufficient to allow fitting. As previously reported [9], an exponential distribution fit the magnitude data extremely well, as shown by the semilog plot in Figure $3 \mathrm{a}$, while for the phase distribution in Figure 3b, a linear dependence fit the data well over the noise free region $(r<6 \mathrm{~m})$. However while the shape of the data fits agree with previous studies [9], the mathematics behind these distributions is expected to be more complicated than those based on only a single thin film or highly thermally conductive film over an insulating substrate. Our thin, highly insulating porous layer over a highly thermally conductive silicon layer leads us to believe the in-plane thermal path (through the porous layer to the silicon) dominates over lateral thermal propagation, altering the solution to the heat equation compared to that obtained for a free standing thin film [5]. As such, an alternative mathematical description of the physics of the problem may be required to explain our data.

3D Temperature magnitude [mK]
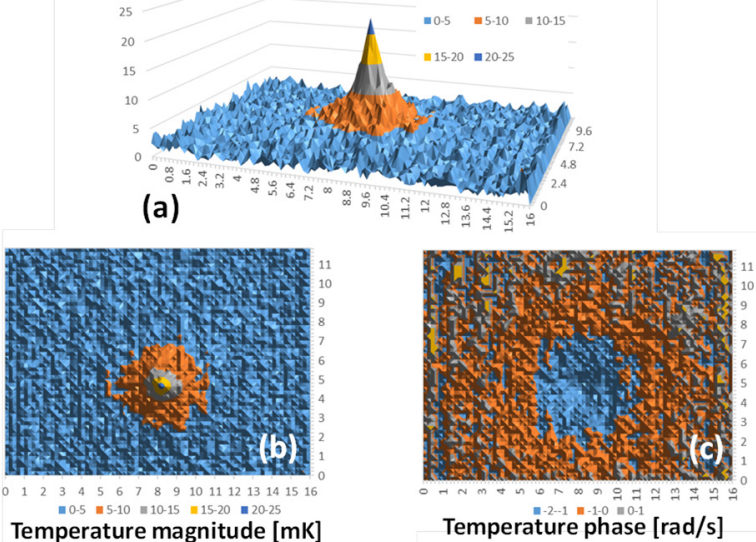

Figure 2: Calibrated (spatially and temperature) thermography image of a porous silicon sample of $84 \%$ porosity heated from a $405 \mathrm{~nm}$ laser modulated at $\boldsymbol{f}_{0}=\mathbf{2 . 5}$ Hz, (a) 3D temperature magnitude showing clear peak, (b) contour temperature magnitude, (c) temperature phase plot.
Nevertheless, across a range of measurements, the fit to an exponential (magnitude) and linear (phase) distribution appears to be very good and allowed us to extract a temperature magnitude diffusion length $\left(\Lambda_{a}\right)$ and a temperature phase diffusion length $\left(\Lambda_{p}\right)$. The models used to define both $\Lambda_{a}$ and $\Lambda_{p}$ are shown inset in Figure 3 along with the fitting parameters for this particular modulation frequency $(f=1 \mathrm{~Hz})$.
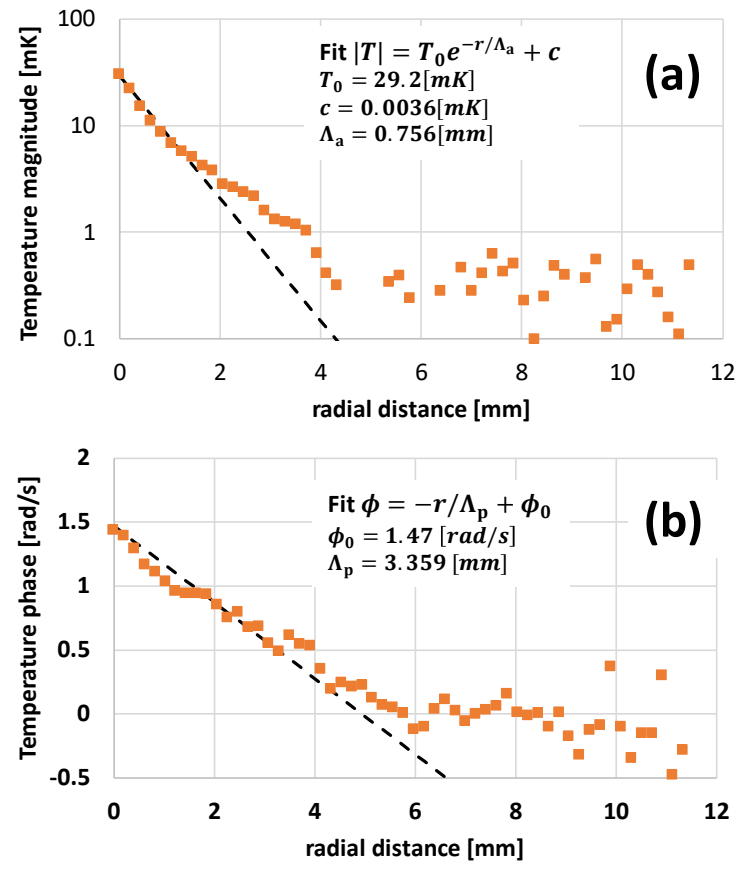

Figure 3: (a) Experimentally obtained radial temperature variation [marker] after a radial histogram average of the data in Figure 2 was performed. Fit (dashed line) using a exponential fit with model (inset) shown over laid, extracting $\Lambda_{a}$, (b) radial variation in thermal phase and linear fit, extracting $\Lambda_{p}$. Measured at $f=1 \mathrm{~Hz}$.

The results of extracting both $\Lambda_{p}$ and $\Lambda_{a}$ parameters across a range of laser modulation frequencies $(f)$ are plotted in Figure $4 a$ and $4 b$, respectively, as a function of $1 / \sqrt{f}$ to highlight the dependency on the thermal diffusion length $L_{D}=\sqrt{D / \pi f}$. For each dataset, the uncertainties were also plotted representing a $95 \%$ confidence interval, obtained using a jackknife analysis of the fitting process [10]. As indicated, the uncertainties in the phase diffusion length $\Lambda_{p}$ are the most significant and more investigation is required to determine the source of these uncertainties. By combining both $\Lambda_{p}$ and $\Lambda_{a}$ we can find the thermal diffusion length [9] which is related to the diffusivity $\mathrm{D}$, by:

$$
\text { thermal diffusion length }=\sqrt{\Lambda_{p} \Lambda_{a}}=L_{D}=\sqrt{D /(\pi f)}
$$

The thermal diffusion length is plotted in Figure $4 \mathrm{c}$ and shows only a weak dependence on $1 / \sqrt{f}$, largely due to the uncertainties introduced by the phase diffusion length, $\Lambda_{p}$. Further the data was obtained only over a limited range of frequencies. By reducing the fitting uncertainties and measuring the thermal maps down to lower frequencies, we expect to significantly improve these measurements. Nevertheless, using the fitted data in Figure 4c, we determined the diffusivity to be $D=1.19 \mathrm{~mm}^{2} / \mathrm{s}$ for this porous silicon sample. Using estimated values for the thermal conductivity of porous silicon and the heat capacity 
of silicon (reduced by the density of porous silicon) shown in Table 1, we estimated the diffusivity of our sample as $D^{*}=2.34 \mathrm{~mm}^{2} / \mathrm{s}$. This value is within a factor of 2 of the value calculated $D$ obtained from our thermography system. Given the issues with the uncertainties and limited frequency range tested, we believe this system is an excellent platform to study and map the thermal properties of porous silicon.

\section{Conclusion}

Through the development of a new tool, we have been able to explore thermography techniques applied to thin insulating porous films over highly thermally conducting silicon substrates. We have demonstrated the ability to detect thermographic images of sufficient signal to noise to be able to undertake parameter extraction and study the dependence on the modulation frequency. Our preliminary estimate for diffusivity of $\mathrm{D}=1.18 \mathrm{~mm}^{2} / \mathrm{s}$ shows good agreement with the expected low diffusivity from these films. However an alternative solution to the heat equation is required to better represent the physics of the problem, and an understanding of the measurement errors is required to improve the thermal parameter estimates. This is a powerful tool that requires further study to realise the full potential of this novel investigative technique.

\section{Acknowledgements}

This work has been supported by the Australian Research Council under DP170104266. We acknowledge the support from the Western Australian Node of the Australian National Fabrication Facility, and the Office of Science of the WA State Government.

\section{References}

[1] A. M. Rossi, G. Amato, V. Camarchia, L. Boarino, and S. Borini, Applied Physics Letters, vol. 78, no. 20, pp. 3003-3005, 2001.

[2] R. Ilaria, M. Antigone, I. Mario, C. Giuseppe, R. Ivo, and S. Luca De, Journal of Physics: Condensed Matter, vol. 20, no. 36, p. 365203, 2008.

[3] L. Meifang, P. Gia, D. John, L. Yinong, and K. Adrian, physica status solidi c, vol. 8, no. 6, pp. 1847-1850, 2011.

[4] A. Ould-Abbas, M. Bouchaour, and N.-E. C. Sari, Open Journal of Physical Chemistry, vol. Vol.02No.01, p. 6, 2012, Art. no. 17521.

[5] O. Breitenstein, W. Warta, and M. Langenkamp, Lockin Thermography: Basics and Use for Evaluating Electronic Devices and Materials. Springer Berlin Heidelberg, 2010.

[6] G. Gesele, J. Linsmeier, V. Drach, J. Fricke, and R. Arens-Fischer, Journal of Physics D: Applied Physics, vol. 30, no. 21, p. 2911, 1997.

[7] Y. S. Touloukian, R. W. Powell, C. Y. Ho, and P. G. Klemens, Thermophysical Properties of Matter - The TPRC Data Series. Volume 1. Thermal Conductivity Metallic Elements and Alloys. Defense Technical Information Center, 1970.

[8] S. Hilmar, B. Otwin, and W. Jan-Martin, physica status solidi (b), vol. 248, no. 9, pp. 2128-2141, 2011.

[9] A. Wolf, P. Pohl, and R. Brendel, Journal of Applied Physics, vol. 96, no. 11, pp. 6306-6312, 2004.

[10] B. Efron, "The Jackknife, the Bootstrap, and other Resampling Plans," Division of Biostatistics, Stanford University, Stanford UniversityDecember, 19801980.
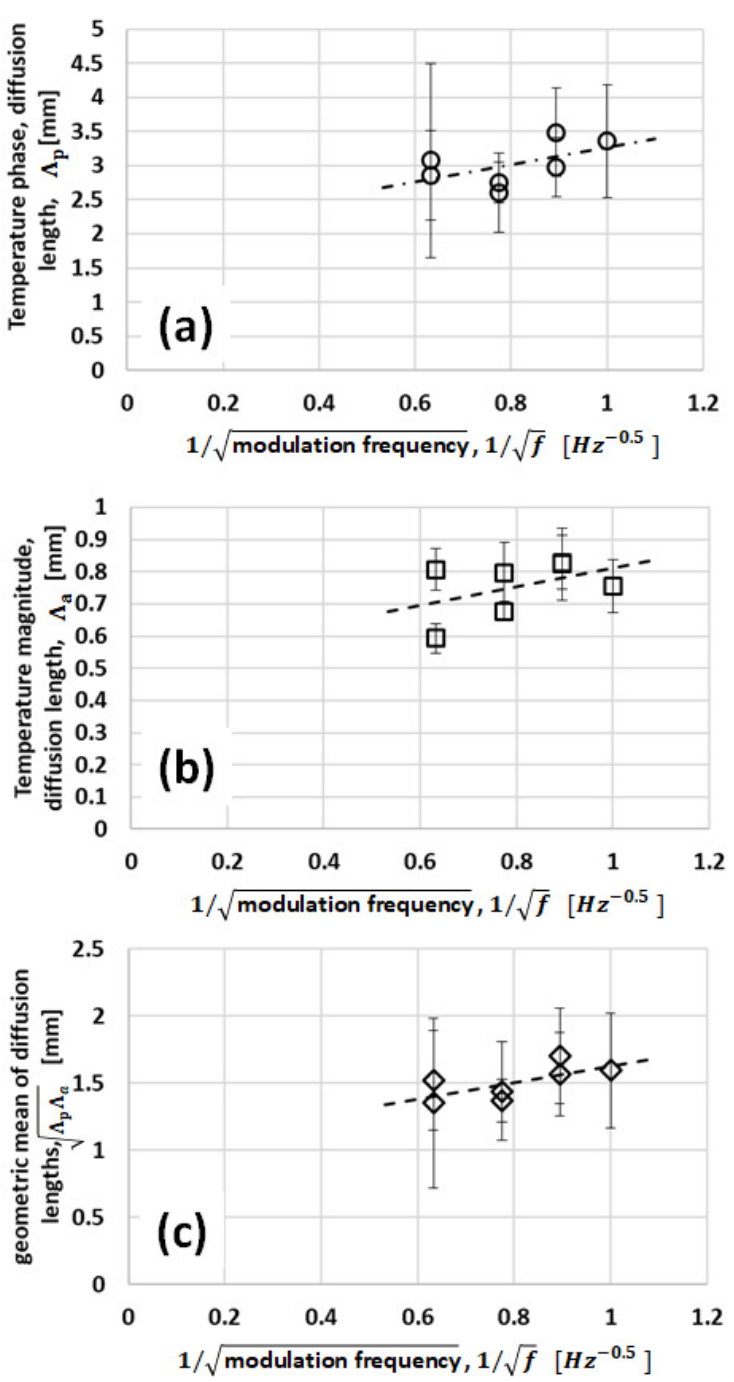

Figure 4: Parameters plotted as a function of $\frac{1}{\sqrt{f}}$ to determine dependence on diffusivity: (a) extracted phase diffusion length, $\Lambda_{p}$; (b) extracted magnitude diffusion length, $\Lambda_{a}$; (c) combined thermal (geometric) diffusion length, $\sqrt{\Lambda_{a} \Lambda_{p}}$. Uncertainties with a $95 \%$ confidence were obtained from a jackknife analysis of the least squares fit. Dashed lines are lines of best fit to the data.

Table 1: Estimated thermal parameters for porous silicon

\begin{tabular}{lrl} 
Parameter & Value & Units \\
Porosity & $84 \%$ & \\
\hline Thermal Conductivity & 0.61 & $\mathrm{~W} / \mathrm{m} . \mathrm{K}$ \\
\hline Density & 0.37 & $\mathrm{gm} / \mathrm{cm}^{3}$ \\
Specific Heat Capacity & 0.71 & $\mathrm{~J} / \mathrm{gm} . \mathrm{K}$ \\
\hline Diffusivity & 2.34 & $\mathrm{~mm} 2 / \mathrm{s}$
\end{tabular}

